Pacific Journal of Mathematics

EXISTENCE OF DIRICHLET FINITE BIHARMONIC
FUNCTIONS ON THE POINCARE 3-BALL 


\title{
EXISTENCE OF DIRICHLET FINITE BIHARMONIC FUNCTIONS ON THE POINCARÉ 3-BALL
}

\author{
Leo Sario and Cecilia WaNg
}

In an earlier study we discussed the existence of quasiharmonic functions, i.e., solutions of $\Delta u=1$. We showed, in particular, that there exist Dirichlet finite quasiharmonic functions on the Poincaré 3-ball

$$
B_{\alpha}:\left\{|x|<1, d s=\left(1-|x|^{2}\right)^{\alpha}|d x|\right\}
$$

if and only if $\alpha \in(-3 / 5,1)$. We now ask: Is the existence of these functions entailed by that of Dirichlet biharmonic functions? This is known to be the case for dimension 2 . We shall show that, perhaps somewhat unexpectedly, it is no longer true for dimension 3 .

For preparation we first solve the problem, of significance in its own right, of the existence of Dirichlet finite biharmonic functions. In the notation of No. 1 below, we give the complete characterization

$$
B_{\alpha} \notin O_{H^{2} D} \Longleftrightarrow \alpha>-\frac{3}{5}
$$

The problem also offers considerable technical interest, as the generating harmonic functions can not be presented in a closed form, but only by means of expansions at the regular singular point of the related differential equation. This makes the estimates somewhat delicate. Also, the four cases $\alpha \geqq 1, \alpha \in(-3 / 5,1), \alpha<-3 / 5$, and $\alpha=-3 / 5$ must be treated separately, each with its own approach.

To deduce the above result (Theorem 1), we first expand a harmonic function on $B_{\alpha}$ in terms of spherical harmonics with respect to our non-Euclidean metric (Theorem 2). As important applications of Theorem 1 to the classification theory we obtain a decomposition of the totality of Riemannian 3-manifolds into three disjoint nonempty subclasses induced by $O_{Q D}$ and $O_{H^{2} D}$ (Theorem 3), and establish the existence of parabolic 3-manifolds which carry $H^{2} D$-functions and of hyperbolic 3-manifolds which do not carry $H^{2} D$-functions (Theorem 4).

An interesting open problem is whether $B_{\alpha} \notin O_{H^{2} D}$ if and only if $\alpha>-3 /(N+2)$.

1. A function $u$ is harmonic or biharmonic according as it satisfies $\Delta_{\lambda} u=0$ or $\Delta_{\lambda}^{2} u=0$, where $\Delta_{2}$ is the Laplace-Beltrami operator $\Delta_{2}=$ $d \delta+\delta d$ with respect to the metric $d s=\lambda(x)|d x|$. Denote by $H^{2}$ the family of nonharmonic biharmonic functions, by $D$ the family of 
functions $f$ with finite Dirichlet integrals $D(f)=\int d f \wedge * d f<\infty$, and set $H^{2} D=H^{2} \cap D$. Let $O_{H^{2} D}$ be the class of Riemannian manifolds which do not carry $H^{2} D$-functions. We assert:

THEOREM 1. $B_{\alpha} \notin O_{H^{2} D} \Leftrightarrow \alpha>-3 / 5$.

The proof will be given in Nos. 2-7.

2. We start by expanding a harmonic function on $B_{\alpha}$ in spherical harmonics. We recall that a function $S_{n}\left(\theta^{1}, \theta^{2}\right)$, in polar coordinates $\left(r, \theta^{1}, \theta^{2}\right)$, is called a spherical harmonic of degree $n$ if $r^{n} S_{n}\left(\theta^{1}, \theta^{2}\right)$ is harmonic with respect to the Euclidean metric. Every such function is a unique linear combination of $2 n+1$ linearly independent fundamental spherical harmonics $S_{n m}$ of degree $n$. The class $\left\{S_{n m} ; n=\right.$ $0,1,2, \cdots ; m=1,2, \cdots, 2 n+1\}$ is not only an orthogonal system with respect to the inner product $(f, g)=\int_{\omega} f g d S$, with $\omega$ the 2-sphere and $d S$ the surface element, but also a complete system with respect to the family of $L^{2}$-functions. For every harmonic function $h$ in $B_{\alpha}$, we have a Fourier expansion

$$
h(r, \theta)=\sum_{n=0}^{\infty} \sum_{m=1}^{2 n+1} d_{n m}(r) S_{n m}(\theta)
$$

with $\theta=\left(\theta^{1}, \theta^{2}\right)$.

By virtue of

$$
\Delta_{\lambda}\left(f(r) S_{n m}(\theta)\right)=-\lambda^{-2}\left[f^{\prime \prime}(r)+\left(\frac{2}{r}+\frac{\lambda^{\prime}}{\lambda}\right) f^{\prime}(r)-n(n+1) r^{-2} f(r)\right] S_{n m}(\theta),
$$

and $\lambda^{\prime} \lambda^{-1}=-2 \alpha r\left(1-r^{2}\right)^{-1}$, the function $f(r) S_{n m}(\theta)$ is harmonic on $B_{\alpha}$ if and only if $f(r)$ satisfies the differential equation

$$
\begin{aligned}
r^{2}(1 & \left.-r^{2}\right) f^{\prime \prime}(r)+r\left[2\left(1-r^{2}\right)-2 \alpha r^{2}\right] f^{\prime}(r) \\
& -n(n+1)\left(1-r^{2}\right) f(r)=0 .
\end{aligned}
$$

We shall denote the solution of equation (2) for each $n$ by $f_{n}(r)$. Since all coefficients in (2) can be expanded into power series of $r$, the point 0 is a regular singular point of the equation. Thus there exists at least one solution of (2) in the form

$$
f_{n}(r)=r^{p_{n}} \sum_{i=0}^{\infty} c_{n i} r^{i}
$$

$c_{n 0} \neq 0$. On substituting in (2) we have 


$$
\begin{aligned}
& \sum_{i=0}^{\infty}\left[\left(p_{n}+i-1\right)\left(p_{n}+i\right)+2\left(p_{n}+i\right)-n(n+1)\right] c_{n i} r^{p_{n}+i} \\
& -\sum_{i=2}^{\infty}\left\{\left(p_{n}+i-3\right)\left(p_{n}+i-2\right)+(2+2 \alpha)\left(p_{n}+i-2\right)\right. \\
& -n(n+1)\} c_{n, i-2} r^{p_{n}+i}=0 .
\end{aligned}
$$

To determine $p_{n}$ we equate to 0 the coefficient of $r^{p_{n}}$ and obtain the indicial equation

$$
\left(p_{n}-1\right) p_{n}+2 p_{n}-n(n+1)=0
$$

which gives $p_{n}=n$ or $p_{n}=-(n+1)$. Since $0 \in B_{\alpha}, p_{n}$ can not be negative, and therefore $p_{n}=n$.

We then equate to 0 the coefficient $2(n+1) c_{n 1}$ of $r^{p_{n}+1}$ and obtain $c_{n 1}=0$.

To find $c_{n i}, i \geqq 2$, we equate to 0 the coefficient of $r^{p_{n}+i}$ :

$$
\begin{aligned}
& {\left[\left(p_{n}+i\right)\left(p_{n}+i+1\right)-n(n+1)\right] c_{n i} } \\
= & {\left[\left(p_{n}+i-2\right)\left(p_{n}+i-1+2 \alpha\right)-n(n+1)\right] c_{n, i-2} . }
\end{aligned}
$$

On letting $p_{n}=n$ and $c_{n 0}=1$ we have

$$
c_{n, 2 i}=\prod_{j=1}^{i} \frac{(n+2 j-2)(n+2 j-1+2 \alpha)-n(n+1)}{(n+2 j)(n+2 j+1)-n(n+1)}
$$

for $i \geqq 1$, and $c_{n, 2 i+1}=0$ for $i \geqq 0$.

The limit of $f_{n}(r)=\sum_{i=0}^{\infty} c_{n, 2 i} r^{n+2 i}$ as $r \rightarrow 1$ exists since the $c_{n, 2 i}$ are of constant sign as soon as $i$ is sufficiently large. Furthermore, this limit can not be zero, for otherwise $\lim _{r \rightarrow 1} f_{n} S_{n m} \equiv 0$, and consequently $f_{n} \equiv 0$, contrary to $c_{n 0}=1$. In a similar fashion we see that $f_{n}(r) \neq 0$ for $0<r<1$. Hence for arbitrary but fixed $r_{0}, 0<r_{0}<1$, there exist constants $a_{n m}$ such that $a_{n m} f_{n}\left(r_{0}\right) S_{n m}=d_{n m}\left(r_{0}\right) S_{n m}$, and

$$
\sum_{n=0}^{\infty} \sum_{m=1}^{2 n+1} a_{n m} f_{n}(r) S_{n m}(\theta)
$$

is a series of functions harmonic on $B_{\alpha}$ which converges absolutely and uniformly to $h\left(r_{0}, \theta\right)$ on the 2-sphere of radius $r_{0}$. Now let $r_{0}<r^{\prime}<1$; then by the same argument there exist constants $a_{n m}^{\prime}$ such that

$$
\sum_{n=0}^{\infty} \sum_{m=1}^{2 n+1} a_{n m}^{\prime} f_{n}(r) S_{n m}(\theta)
$$

converges to $h$ on the ball of radius $r^{\prime}$. Hence (6) and (7) are identical on the ball of radius $r_{0}$, so that $a_{n m}=a_{n m}^{\prime}$ for all $(n, m)$.

We have proved: 
THEOREM. Every harmonic function $h\left(r, \theta^{1}, \theta^{2}\right)$ on the Poincare ball $B_{\alpha}$ has the expansion in terms of the fundamental spherical harmonics $S_{n m}$,

$$
h\left(r, \theta^{1}, \theta^{2}\right)=\sum_{n=0}^{\infty} \sum_{m=1}^{2 n+1} a_{n m} \sum_{i=0}^{\infty} c_{n, 2 i} r^{n+2 i} S_{n m}\left(\theta^{1}, \theta^{2}\right),
$$

where the $c_{n, 2 i}$ are given by (5).

3. After this preparation, we proceed with the proof of Theorem 1. An essential aspect of the proof is that the cases $\alpha \geqq 1, \alpha \in$ $(-3 / 5,1), \alpha<-3 / 5$, and $\alpha=-3 / 5$ all require a different treatment.

We first establish the following crucial estimate:

Lemma 1. If $\alpha \geqq 1$, then

$$
f_{1}(r)=\sum_{i=0}^{\infty} c_{1,2 i} r^{1+2 i}=O\left((1-r)^{-2 \alpha}\right) \text { as } r \longrightarrow 1 \text {. }
$$

Proof. By (5),

$$
\begin{aligned}
c_{1,2 i} & =\prod_{j=1}^{i} \frac{(2 j-1)(2 j+2 \alpha)-2}{(2 j+1)(2 j+2)-2} \\
& =\prod_{j=1}^{i} \frac{2 j+2 \alpha-1}{2 j} \cdot \frac{2 j-1+(2 j-3) /(2 j+2 \alpha-1)}{2 j+3} .
\end{aligned}
$$

We claim that

$$
c_{1,2 i}<\prod_{j=1}^{i} \frac{2 j+2 \alpha-1}{2 j}
$$

or, equivalently,

$$
4-\frac{2 j-3}{2 j+2 \alpha-1}>0 .
$$

In the case $\alpha \geqq 1$ under consideration this is clearly so for all $j \geqq 1$. Consequently

$$
f_{1}(r)<r+\sum_{i=1}^{\infty}\left(\prod_{j=1}^{i} \frac{2 j+2 \alpha-1}{2 j}\right) r^{1+2 i} .
$$

We compare this with the expansion

$$
\begin{aligned}
r(1-r)^{-2 \alpha} & =r+\sum_{i=1}^{\infty}\left(\prod_{j=1}^{i} \frac{j+2 \alpha-1}{j}\right) r^{1+i} \\
& >r+\sum_{i=1}^{\infty}\left(\prod_{j=1}^{i} \frac{2 j+2 \alpha-1}{2 j}\right) r^{1+2 i}
\end{aligned}
$$

and obtain the lemma. 
4. We shall make use of Lemma 1 to prove:

LEMMA 2. $B_{\alpha} \notin O_{H^{2} D}$ for $\alpha \geqq 1$.

Proof. A necessary and sufficient condition for the existence of an $H^{2} D$-function $u$ is that the Laplacian $\Delta u=h$ satisfies

$$
|(h, \varphi)| \leqq K \sqrt{D(\varphi)}
$$

for all $\varphi \in C_{0}^{\infty}$ and some constant $K$ independent of $\varphi$ (Nakai-Sario [5]).

Let $h=f_{1}(r) S_{11}=f_{1}(r) \cos \theta^{1}$, and take any $\varphi \in C_{0}^{\infty}\left(B_{\alpha}\right)$. By Lemma 1 and the Fourier expansion

$$
\varphi=\sum_{n=0}^{\infty} \sum_{m=1}^{2 n+1} b_{n m}(r) S_{n m}\left(\theta^{1}, \theta^{2}\right),
$$

we obtain

$$
\begin{aligned}
|(h, \varphi)| & =\mid \text { const } \int_{0}^{1} b_{11}(r) f_{1}(r) r^{2}\left(1-r^{2}\right)^{3 \alpha} d r \mid \\
& <\text { const } \int_{0}^{1}\left|b_{11}(r)\right|(1-r)^{\alpha} d r .
\end{aligned}
$$

By Schwarz's inequality,

$$
\begin{aligned}
|(h, \varphi)|^{2} & \leqq \text { const } \int_{0}^{1}(1-r)^{\alpha} d r \cdot \int_{0}^{1} b_{11}^{2}(r)(1-r)^{\alpha} d r \\
& =\operatorname{const} \int_{0}^{1} b_{11}^{2}(r)(1-r)^{\alpha} d r .
\end{aligned}
$$

On the other hand,

$$
D(\varphi)
$$

$=\int_{B_{\alpha}}|\operatorname{grad} \varphi|^{2} d V \geqq$ const $\int_{B_{\alpha}} r^{-2}\left(1-r^{2}\right)^{-2 \alpha}\left(\frac{\partial \varphi}{\partial \theta^{1}}\right)^{2} r^{2}\left(1-r^{2}\right)^{3 \alpha} d r$

$$
\geqq \text { const } \int_{0}^{1} b_{11}^{2}(r)(1-r)^{\alpha} d r \text {. }
$$

5. Denote by $Q$ the class of quasiharmonic functions $u$, characterized by $\Delta_{\lambda} u=1$. We recall (Sario-Wang [9]) that $B_{\alpha} \notin O_{Q D}$ if and only if $\alpha \in(-3 / 5,1)$. Since $Q D \subset H^{2} D$, we have trivially:

Lemma 3. $B_{\alpha} \notin O_{H^{2} D}$ if $\alpha \in(-3 / 5,1)$.

6. Next we consider the case $\alpha<-3 / 5$.

Lemma 4. $B_{\alpha} \in O_{H^{2} D}$ if $\alpha<-3 / 5$.

Proof. Suppose there exists an $H^{2} D$-function $u$ on $B_{\alpha}$, that is, 
$\Delta u=h$ satisfies (11). By Theorem $2, h$ has the expansion

$$
h=\sum_{n=0}^{\infty} \sum_{m=1}^{2 n+1} \sum_{i=0}^{\infty} a_{n m} c_{n, 2 i} r^{n+2 i} S_{n m} .
$$

If $a_{n m} \neq 0$ for some $(n, m)$, choose for our testing functions $\varphi_{t}$, $0<t \leqq 1$,

$$
\varphi_{t}(r, \theta)=\rho_{t}(r) S_{n m}(\theta), \quad \rho_{t}(r)=g\left(\frac{1-r}{t}\right)
$$

where $g(r)$ is a fixed nonnegative $C_{0}^{\infty}$-function with supp $g \subset(\beta, \gamma)$, $0<\beta<\gamma<1$. Since $\lim _{r \rightarrow 1} f_{n}(r) \neq 0$,

$$
\int_{1-\gamma t}^{1-\beta t} \rho_{t}(r) d r=t \int_{\beta}^{r} g(r) d r,
$$

and $\left(1-r^{2}\right)^{3 \alpha}>2^{3 \alpha}(1-r)^{3 \alpha} \geqq 2^{3 \alpha}(\gamma t)^{3 \alpha}$ for $\alpha<0$, we have for sufficiently small $t$,

$$
\begin{aligned}
& \left|\left(h, \varphi_{t}\right)\right|=\mathrm{const}\left|\int_{1-\gamma t}^{1-\beta t} f_{n}(r) \rho_{t}(r) r^{2}\left(1-r^{2}\right)^{3 \alpha} d r\right| \\
& \geqq \text { const }(1-\gamma)^{2}(\gamma t)^{3 \alpha} \int_{1-\gamma t}^{1-\beta t} \rho_{t}(r) d r=\text { const } t^{3 \alpha+1} .
\end{aligned}
$$

On the other hand,

$$
\begin{aligned}
D\left(\varphi_{t}\right) & =\int_{B_{\alpha}}\left|\operatorname{grad} \varphi_{t}\right|^{2} d V \\
& =\int_{1-\gamma t}^{1-\beta t}\left(1-r^{2}\right)^{-2 \alpha}\left(c_{1}\left(\rho^{\prime}(r)\right)^{2}+c_{2} r^{-2} \rho^{2}(r)\right) r^{2}\left(1-r^{2}\right)^{3 \alpha} d r \\
& <\operatorname{const}(\gamma t)^{\alpha} \int_{1-\gamma t}^{1-\beta t}\left(c_{1}\left(\rho^{\prime}(r)\right)^{2}+c_{2} \rho^{2}(r)\right) d r \\
& =d_{1} t^{\alpha-1}+d_{2} t^{\alpha+1}<d t^{\alpha-1},
\end{aligned}
$$

$\alpha<0$, where $d_{1}, d_{2}$, and $d$ are independent of $t$. If $\alpha<-3 / 5$, then (11) is violated as $t \rightarrow 0$, a contradiction. Thus $B_{\alpha} \in O_{H^{2} D}$ for $\alpha<-3 / 5$.

7. It remains to consider the case $\alpha=-3 / 5$.

Lemia 5. $B_{-3 / 5} \in O_{H H^{2} D}$.

Proof. We choose a decreasing sequence of real numbers $t_{j} \in(0,1]$ tending to 0 such that $1-\beta t_{j}<1-\gamma t_{j+1}$ and (14) is satisfied for each $t_{j}$. Set $q_{j}=t_{j}^{-3 \alpha-1} j^{-1} \cdot \operatorname{sign}\left(h, \varphi_{t_{j}}\right)$ and take for the testing functions $\varphi_{n}=\sum_{j=1}^{n} q_{j} \varphi_{t_{j}}$. We obtain by (14)

$$
\left|\left(h, \varphi_{n}\right)\right|=\left|\sum_{j=1}^{n} q_{j}\left(h, \varphi_{t_{j}}\right)\right|>\operatorname{const} \sum_{j=1}^{n} j^{-1}
$$


and by (15)

$$
D\left(\varphi_{n}\right)=\sum_{j=1}^{n} q_{j}^{2} D\left(\varphi_{t_{j}}\right)<\operatorname{const} \sum_{j=1}^{n} j^{-2}\left(t_{j}^{-5 \alpha-3}\right) .
$$

For $\alpha=-3 / 5$, we have $D\left(\varphi_{n}\right)<$ const $\sum_{1}^{n} j^{-2}$, which stays bounded as $n \rightarrow \infty$ whereas $\left|\left(h, \varphi_{n}\right)\right| \rightarrow \infty$. Thus (11) is violated, and we conclude that $B_{-3 / 5} \in O_{H^{2} D}$.

The proof of Theorem 1 is herewith complete.

8. Since $B_{\alpha} \notin O_{Q D}$ if and only if $\alpha \in(-3 / 5,1)$, Theorem 1 has the following applications to the classification of Riemannian manifolds, with $\widetilde{O}$ standing for the complement of $O$ :

THEOREm 3. The totality of Riemannian 3-manifolds has the decomposition

$$
\{R\}=O_{H^{2} D} \cup\left(O_{Q D} \cap \widetilde{O}_{H^{2} D}\right) \cup \widetilde{O}_{Q D}
$$

into three disjoint nonempty subclasses.

THEOREM 4. There exist parabolic Riemannian 3-manifolds which carry $H^{2} D$-functions, and hyperbolic Riemannian 3-manifolds which do not carry $H^{2} D$-functions.

For dimension 2, this was shown in Nakai-Sario [5], but for higher dimensions it has been an open problem.

For the proof of Theorem 4, let $O_{G}$ be the class of parabolic Riemannian manifolds. It was proved in Sario-Wang [9] that $B_{\alpha} \in O_{G}$ if and only if $\alpha \geqq 1$. As a consequence,

$$
\begin{aligned}
& B_{\alpha} \in O_{G} \cap \widetilde{O}_{H^{2} D} \Longleftrightarrow \alpha \geqq 1, \\
& B_{\alpha} \in \widetilde{O}_{G} \cap O_{H^{2} D} \Longleftarrow \alpha \leqq-\frac{3}{5} .
\end{aligned}
$$

We shall return to the classification of higher dimensional Riemannian manifolds in further studies.

We are indebted to Mr. Dennis Hada, who preused the manuscript and made his valued comments.

\section{REFERENCES}

1. Y. K. Kwon, L. Sario, and B. Walsh, Behavior of biharmonic functions on Wiener's and Royden's compactifications, Ann. Inst. Fourier, (Grenoble), 21 (1971), 217-226.

2. C. Müller, Spherical Harmonics, Lecture Notes in Mathematics 17, Springer, 1966. 
3. M. Nakai and L. Sario, Biharmonic classification of Riemannian manifolds, Bull. Amer. Math. Soc., 77 (1971), 432-436.

4. - Quasiharmonic classification of Riemannian manifolds, Proc. Amer. Math. Soc., 31 (1972), 165-169.

5. - Existence of Dirichlet finite biharmonic functions, Ann. Acad. Sci. Fenn. A. I. 532 (1973), 1-33.

6. H. O'Malla, Dirichlet finite biharmonic functions on the unit disk with distorted metrics, Proc. Amer. Math. Soc., 32 (1972), 521-524.

7. R. Narasimhan, Analysis on Real and Complex Manifolds, Masson, Paris, 1968, $246 \mathrm{pp}$.

8. L. Sario, Biharmonic and quasiharmonic functions on Riemannian manifolds, Duplicated lecture notes 1969-1970, University of California, Los Angeles.

9. L. Sario and C. Wang, Quasiharmonic functions on the Poincaré $\mathrm{N}$-ball, Rend. Mat., (to appear).

Received July 19, 1972. The work was sponsored by the U. S. Army Research Office-Durham, Grant DA-ARO-D-31-124-71-G181, University of California, Los Angeles.

UNiversity of CALIFornia, Los ANGELeS 


\section{PACIFIC JOURNAL OF MATHEMATICS}

\section{EDITORS}

RICHARD ARENS (Managing Editor)

University of California

Los Angeles, California 90024

R. A. Beaumont

University of Washington

Seattle, Washington 98105
J. DUGUNDJI*

Department of Mathematics

University of Southern California

Los Angeles, California 90007

D. Gilbarg and J. Milgram

Stanford University

Stanford, California 94305

\section{ASSOCIATE EDITORS}
E. F. BECKENBACH
B. H. NeumanN
F. WOLF
K. YosHIDA

\section{SUPPORTING INSTITUTIONS}

UNIVERSITY OF BRITISH COLUMBIA
CALIFORNIA INSTITUTE OF TECHNOLOGY
UNIVERSITY OF CALIFORNIA
MONTANA STATE UNIVERSITY
UNIVERSITY OF NEVADA
NEW MEXICO STATE UNIVERSITY
OREGON STATE UNIVERSITY
UNIVERSITY OF OREGON
OSAKA UNIVERSITY

UNIVERSITY OF BRITISH COLUMBIA

UNIVERSITY OF CALIFORNIA

MONTANA STATE UNIVERSITY

UNIVERSITY OF NEVADA

OREGON STATE UNIVERSITY

OSAKA UNIVERSITY
UNIVERSITY OF SOUTHERN CALIFORNIA

STANFORD UNIVERSITY

UNIVERSITY OF TOKYO

UNIVERSITY OF UTAH

WASHINGTON STATE UNIVERSITY

UNIVERSITY OF WASHINGTON

\section{AMERICAN MATHEMATICAL SOCIETY} NAVAL WEAPONS CENTER

* C. R. DePrima California Institute of Technology, Pasadena, CA 91109, will replace J. Dugundji until August 1974. 


\section{Pacific Journal of Mathematics}

\section{Vol. 48, No. $1 \quad$ March, 1973}

Jan Aarts and David John Lutzer, Pseudo-completeness and the product of Baire

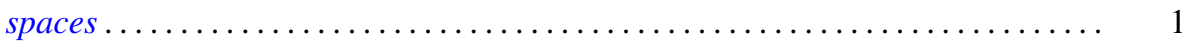

Gordon Owen Berg, Metric characterizations of Euclidean spaces ............ 11

Ajit Kaur Chilana, The space of bounded sequences with the mixed topology ..... . 29

Philip Throop Church and James Timourian, Differentiable open maps of

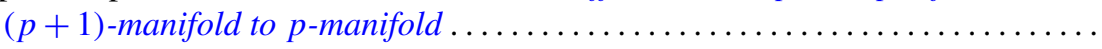

P. D. T. A. Elliott, On additive functions whose limiting distributions possess a finite

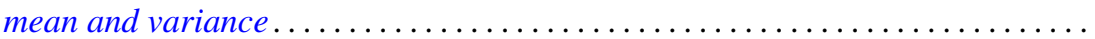

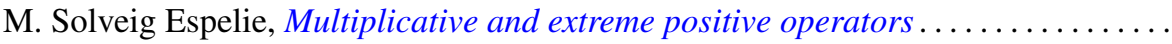

Jacques A. Ferland, Domains of negativity and application to generalized convexity

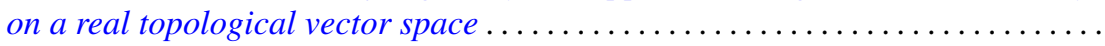

Michael Benton Freeman and Reese Harvey, A compact set that is locally holomorphically convex but not holomorphically convex ...............

Roe William Goodman, Positive-definite distributions and intertwining

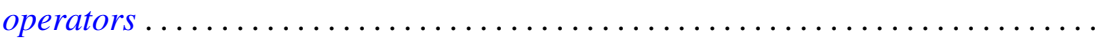

Elliot Charles Gootman, The type of some $C^{*}$ and $W^{*}$-algebras associated with

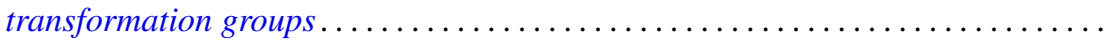

David Charles Haddad, Angular limits of locally finitely valent holomorphic

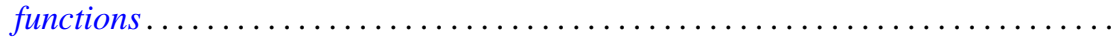

William Buhmann Johnson, On quasi-complements .

William M. Kantor, On 2-transitive collineation groups of finite projective spaces...

Joachim Lambek and Gerhard O. Michler, Completions and classical localizations of right Noetherian rings

Kenneth Lamar Lange, Borel sets of probability measures ......

David Lowell Lovelady, Product integrals for an ordinary differential equation in a Banach space

Jorge Martinez, A hom-functor for lattice-ordered groups .........

W. K. Mason, Weakly almost periodic homeomorphisms of the two sphere ....

Anthony G. Mucci, Limits for martingale-like sequences .......

Eugene Michael Norris, Relationally induced semigroups ...

Arthur E. Olson, A comparison of c-density and $k$-density ......

Donald Steven Passman, On the semisimplicity of group rings of linear groups.

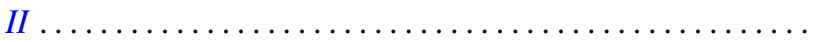

Charles Radin, Ergodicity in von Neumann algebras .

P. Rosenthal, On the singularities of the function generated by the Bergman operator of the second kind.

Arthur Argyle Sagle and J. R. Schumi, Multiplications on homogeneous spaces,

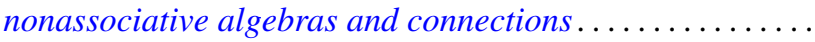

Leo Sario and Cecilia Wang, Existence of Dirichlet finite biharmonic functions on

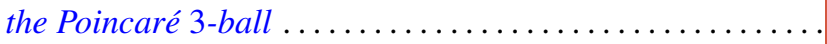

Ramachandran Subramanian, On a generalization of martingales due to Blake ..

Bui An Ton, On strongly nonlinear elliptic variational inequalities.

Seth Warner, A topological characterization of complete, discretely valued

fields. 